\title{
Machine Learning Based Method for Estimating Energy Losses in Large-Scale Unbalanced Distribution Systems with Photovoltaics
}

\author{
Karar Mahmoud ${ }^{1,3}$, Mohamed Abdel-Nasser ${ }^{2,3}$, Heba Kashef ${ }^{3}$, Domenec Puig ${ }^{2}$, Matti Lehtonen ${ }^{1}$ \\ ${ }^{1}$ Department of Electrical Engineering and Automation, Aalto University, Espoo FI-00076 (Finland) \\ ${ }^{2}$ Departament d'Enginyeria Informàtica i Matemàtiques, Universitat Rovira i Virgili, Tarragona 43007 \\ (Spain) \\ ${ }^{3}$ Department of Electrical Engineering, Aswan University, Aswan 81542 (Egypt)
}

Received 11 November 2019 | Accepted 27 July 2020 | Published 6 August 2020

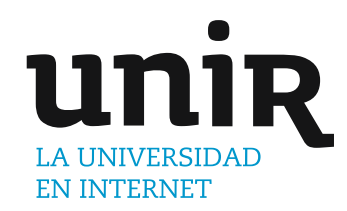

KEYWORDS

Machine Learning, Neural Networks, Energy Loss, Large-Scale Unbalanced Distribution System, Photovoltaics.

DOI: $10.9781 /$ ijimai.2020.08.002

In the recent years, the penetration of photovoltaics (PV) has obviously been increased in unbalanced power distribution systems. Driven by this trend, comprehensive simulation tools are required to accurately analyze large-scale distribution systems with a fast-computational speed. In this paper, we propose an efficient method for performing time-series simulations for unbalanced power distribution systems with PV. Unlike the existing iterative methods, the proposed method is based on machine learning. Specifically, we propose a fast, reliable and accurate method for determining energy losses in distribution systems with PV. The proposed method is applied to a large-scale unbalanced distribution system (the IEEE 906 Bus European LV Test Feeder) with PV grid-connected units. The method is validated using OpenDSS software. The results demonstrate the high accuracy and computational performance of the proposed method.

is based on creating a similar day matrix that is obtained using the statistical analysis of different weather conditions. The impact of the PV system in a low voltage network has been tested through three scenarios [7]. Based on this study, the penetration of the PV system should not exceed $50 \%$ with respect to the total load; otherwise, it will contribute to unbalancing voltage and high network losses. For achieving the lowest penetration of the PV, it should be allocated along with the feeders. The deviation in the maximum power point (MPP) in the grid-connected PV system occurrs due to the loss factors that are caused by the various variations in frequency-voltage, irradiance, DC load, and solar cell characteristics [8]. Indeed, the level of penetration of the PV system greatly affects the system losses. In [9], three tests have been applied to different IEEE systems (13, 30 and 69 bus systems) with four different simulation cases. For reducing the losses, the PV system is placed at the bus that is containing the peak value rather than the average value. Implementing different PVs in the systems can contribute to increased energy losses and voltage fluctuation. In [10], the annual energy losses with variant generators are computed, and the impact of different types of DGs at energy losses is analyzed.

Several methods are used for power flow calculations in distribution systems, e.g. Newton Raphson and Gauss-Seidel methods, which are used for non-linear loss calculations [11]-[13]. A fast and accurate method for loss calculation in balanced distribution systems is discussed in [14], which is based on the machine learning techniques. The model is constructed using the Regression Tree technique for various generations of load profiles. Another machine learning method for state estimation of the system using neural methods is discussed

E-mail address: egnaser@gmail.com 
in [15]. This method is applied to small-scale balanced distribution systems without renewable energy. In spite of the previous machine learning-based methods could overcome the computational burden of the iterative methods, they were applied to small systems.

Modern distribution systems require simulation algorithms for estimating energy losses with renewable energy sources, such as $\mathrm{PV}$. In this paper, we propose a machine learning-based method for performing real-time simulations for unbalanced power distribution systems with PV. In our approach, the losses of the large scale systems have been calculated in a very short time and high accuracy using a neural network model. Unlike the existing iterative methods, the proposed method can deliver accurate results in a very short time. The proposed method is applied to a large-scale unbalanced distribution system (the IEEE 906 Bus European LV Test Feeder) with a PV gridconnected unit

The rest of this paper is organized as follows. Section II explains the proposed methodology. Section III presents the results. Section IV concludes the paper and provides some lines of future work.

\section{Proposed Method}

\section{A. Data Structure and Preparation}

In the proposed method, machine learning algorithms are utilized to model the relationship between the input and its corresponding output. In our case, the inputs are active and reactive power profiles of all loads and PVs for the three-phases in the per-unit scale for different time instants.

The active and reactive power profiles for each phase are expressed by (1), and (2), respectively. The $P_{p h}$ matrix involves the values of active power of all PV units and loads for all time instants $\left\{t_{1} \ldots t_{N}\right\}$ in phase $p h \in\{A, B, C\}$ of the distribution system. While the $Q_{p h}$ matrix involves the values of reactive power of all PV units and loads for all time instants $\left\{t_{1} \ldots t_{N}\right\}$ in phase $p h \in\{A, B, C\}$ of the distribution system. $N L$ and $N_{P V}$ stand for the total numbers of loads and PV units in the distribution system, respectively.

On the other hand, the output of the machine learning algorithm is the power losses for all the branches of the distribution system. If we consider that the output is represented by a matrix $P L_{p h}$ as expressed in (3), each element in this matrix $\left(L_{p h}^{t_{m}}\right)$ represents the total system losses at time instant $t_{m}$ for $p h$ phase of the distribution system.

Note that a power flow tool is required to construct the output matrix $P L_{p h \text { based }}$ on the input $P_{p h}$ and $Q_{p h}$ matrices. Indeed, there are available tools that can be used for loss calculations in distribution systems. The OpenDSS supports all frequency domains that relate to the smart grids with renewable energy systems. To validate our approach, we employed OpenDSS software [16] as a benchmark.

\section{B. Constructing A Machine Learning Model}

Indeed, there are many machine learning algorithms that can be utilized to model the energy losses in distribution systems, for example, regression trees, Gaussian processes, logistic regression, support vector machines, and XGboost. In this paper, we use neural networks because it is simple to use, the availability of the neural network tools with graphical user interface (GUI), allowing reproducibility of the studied cases.

In short, neural networks could deal with complex systems, and so they are widely used in data modelling and statistical analysis [17]. The training process of the network is performed by adjusting the weights and biases until reaching the minimum threshold. The techniques of learning neural networks are based on minimizing errors between the output and the desired target. There are different learning techniques such as feed-forward backpropagation (where the errors are directed back to the network input until achieving the network goal) and cascade forward backpropagation.

Here, we describe the architecture of the neural network model while highlighting the way to train the model. For this purpose, Fig. 1 shows the utilized neural network model for the IEEE 906 Bus European LV Test Feeder. The model includes one hidden layer (10 neurons and one output layer). Note that the dimension of the input is 907, which represents the loads, and the output is the total system losses. We build the model using the feed-forward neural networks with initial parameters. The learning algorithm for training the network is the Levenberg Marqudarable (LM) algorithm [18], [19] that has high efficiency. In the training phase, the actual losses are compared with the model output until the network stopping goal is accomplished (maximum number of 300 epochs, minimum 5\% gradient, $1 \mathrm{e}-3$ goal error). If the set goal is not met, the weights and biases are updated with the learning machine rate until the error is equal or less than the set goal. We use the MATLAB Neural Network Toolbox to construct the neural network model.

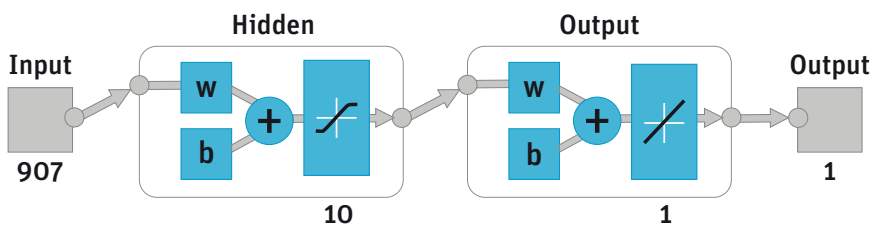

Fig. 1. The architecture of the neural network model (MATLAB 2014a, The MathWorks, Natick, 2014).

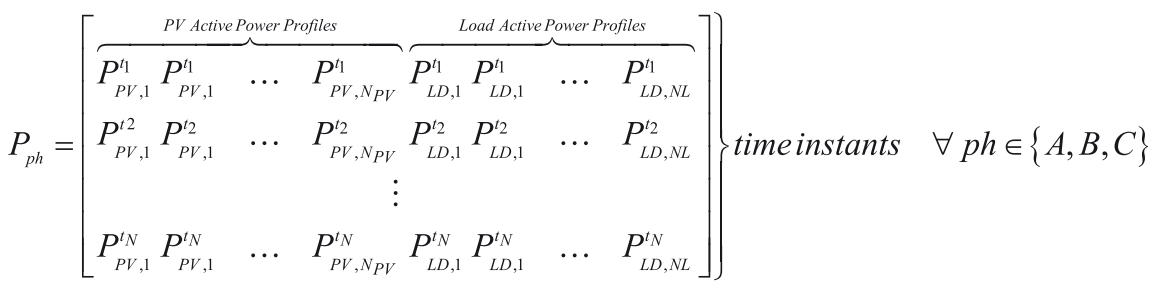

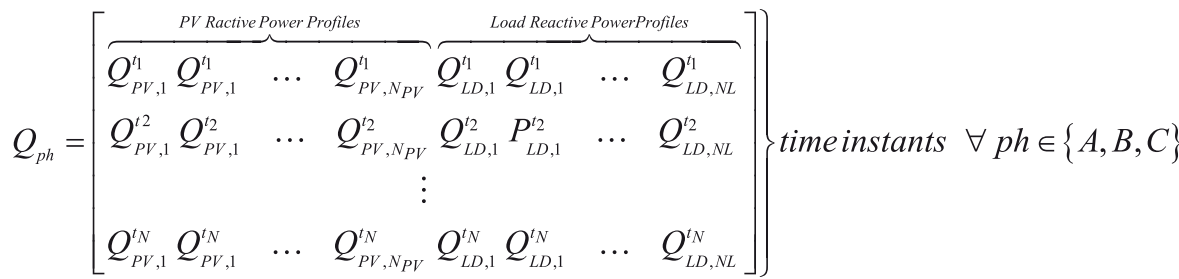

$$
\begin{aligned}
& P L_{p h}=\left[\begin{array}{cccc}
L_{p h}^{t_{1}} & L_{p h}^{t_{2}} & \ldots & L_{p h}^{t_{N}}
\end{array}\right]^{t} \quad \forall p h \in\{A, B, C\}
\end{aligned}
$$




\section{Solution Steps}

Fig. 2 shows the steps for loss estimation using a neural network model in distribution systems with PV. The model is constructed using a synthetic dataset (one-month dataset of loads and PV generation). Specifically, we generate the possible scenarios of loads (43200 load points with 15 minutes time step). Another option is to utilize the reliable forecasting models for PV proposed in [20], [21] to generate the datasets. The corresponding power losses of these datastes are computed offline using OpenDSS. The input (load factors and PV power) and output data (power losses) of the OpenDSS are fed into the neural network to construct the model. Once the training process is completed, the model would be ready for solving the power losses for any input data rapidly and accurately without iterative processes employed in state-of-the-art methods. For testing the proposed model, we use a one-day dataset (1440 samples) at six different resolutions.

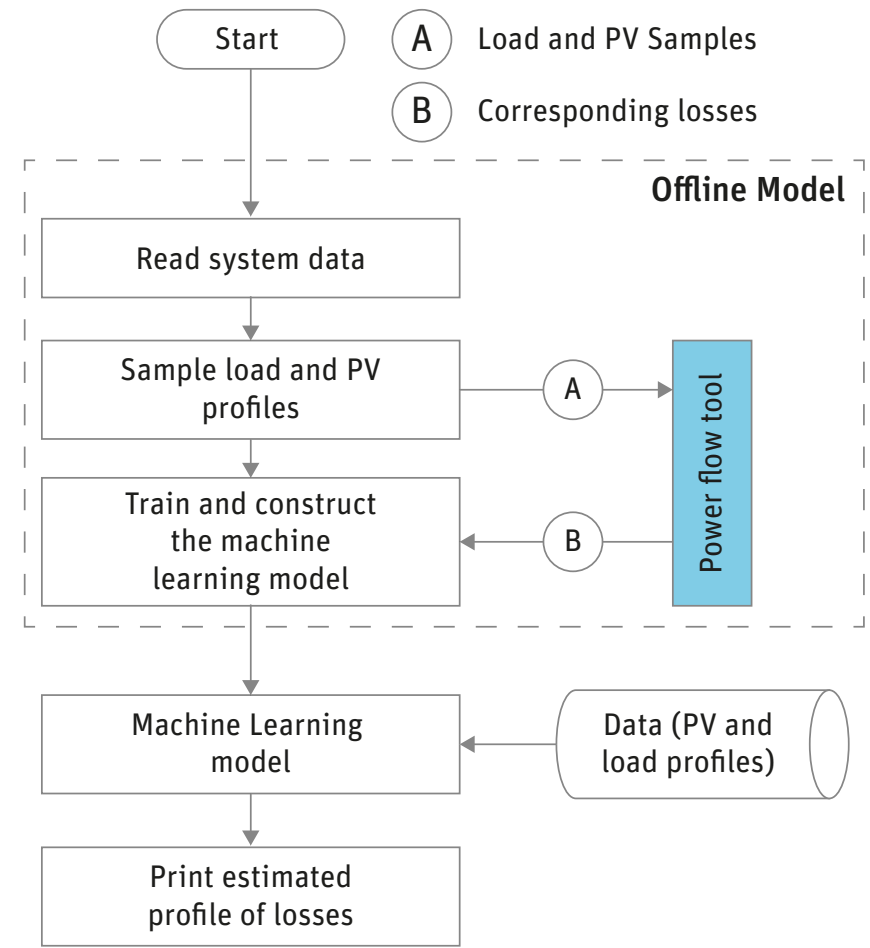

Fig. 2. Flowchart of the proposed method.

Specifically, the following steps are carried out for constructing the offline model and estimating the losses:

a) Read the data of the distribution system interconnected with PV.

b) Read the load and PV states for which the losses are required to be estimated.

c) Generate/read the synthetic dataset and use OpenDSS to calculate the corresponding power losses.

d) Train the neural network and save the model

- Initialize and create a network model.

- Train the created network with its parameters, where the input and the output are being compared until reaching the minimum value of error ( $5 \%$ threshold).

e) Call the model for estimating the losses.

f) The output of the network is simulated and compared to the OpenDSS output for assessing the accuracy of the proposed method. g) Print the analytical and graphical results.

\section{Evaluation Metrics}

The efficacy of the proposed method is quantified by how close the estimated losses are to the exact ones calculated by OpenDSS. Here, different types of errors are computed:

- The mean square error (MSE) in which the average of the squared difference between the estimated and actual values of the power loss is measured by the following formula:

$$
M S E_{p h}={ }_{L}^{1} \sum_{t=t_{1}}^{t_{N}}\left(Y_{p h, t}^{d s s}-Y_{p h, t}^{n n}\right)^{2} \quad \forall p h \in\{A, B, C\}
$$

where $Y_{p h, t}^{d s s}$ and $Y_{p h, t}^{n n}$ are the exact and estimated losses of phase $p h$ at time instant $t$. $L$ represents the number of time instants.

- The root means square error (RMSE), where the square root of the average of the squared values of the difference between the actual and the estimated values are calculated using the following formula:

$$
R M S E_{p h}=\sqrt{\frac{1}{L} \sum_{t=t_{1}}^{t_{m}}\left(\left|X_{p h, t}^{d s s}\right|-\left|X_{p h, t}^{n n}\right|\right)^{2}} \forall p h \in\{A, B, C\}
$$

- The mean absolute error (MAE) where the average difference between the two methods is calculated using the following formula:

$$
M A E_{p h}=\frac{1}{L} \sum_{t=t 1}^{t_{N}}\left(\left|X_{p h, t}^{d s s}-X_{p h, t}^{n n}\right|\right), t=\left\{t_{1}, \ldots . . . t_{m}\right\}, p h \in\{A, B, C\}
$$

- The mean absolute percentage error (MAPE) where the accuracy of the proposed method is expressed as a percentage defined by the following equation:

$$
M A P E_{p h}=\frac{1}{L} \sum_{t=t_{1}}^{t_{N}}\left|\frac{X_{p h, t}^{d s s}-X_{p h, t}^{n n}}{X_{p h, t}^{d s s}}\right| \quad \forall p h \in\{A, B, C\}
$$

- The sum of the squared error (SSE) which is the measure of the scale of variation between the two methods, given by the following equation:

$$
S S E_{p h}=\sum_{i=1}^{n} \sum_{t=t_{1}}^{t_{N}}\left(X_{p h, t}^{d s s}-X_{p h, t}^{n n}\right)^{2} \quad \forall p h \in\{A, B, C\}
$$

- The relative error (RE) which is provided by the following equation:

$$
R E_{p h}=\frac{1}{L} \sum_{t=t_{1}}^{t_{N}} \frac{X_{p h, t}^{d s s}-X_{p h, t}^{n n}}{X_{p h, t}^{d s}} \quad \forall p h \in\{A, B, C\}
$$

All of these errors are calculated for the proposed method to evaluate its accuracy rate compared to the exact one. We have utilized different error formulae (4)-(9) to test the accuracy of the proposed method sufficiently.

\section{RESUlts AND Discussion}

\section{A. Test System and Dataset}

Here, the performance of the proposed method is tested for estimating the energy losses on the MATLAB environment. The results are implemented at Intel ${ }^{\circledR}$ Core $^{\mathrm{TM}}$ i5-5200U CPU @ 2.20GHz, 4.00 GB RAM, and 64-bit Operating System. The IEEE European low voltage test feeder [22] with 907 bus and $50 \mathrm{~Hz}$ frequency (Fig. 3) is used to validate the accuracy and the computational efficiency of the proposed method. Two PV units are connected at busses 639 and 906, and 55 single-phase loads with different daily load shapes (1400 loads points per minute step) are distributed along with the system. 


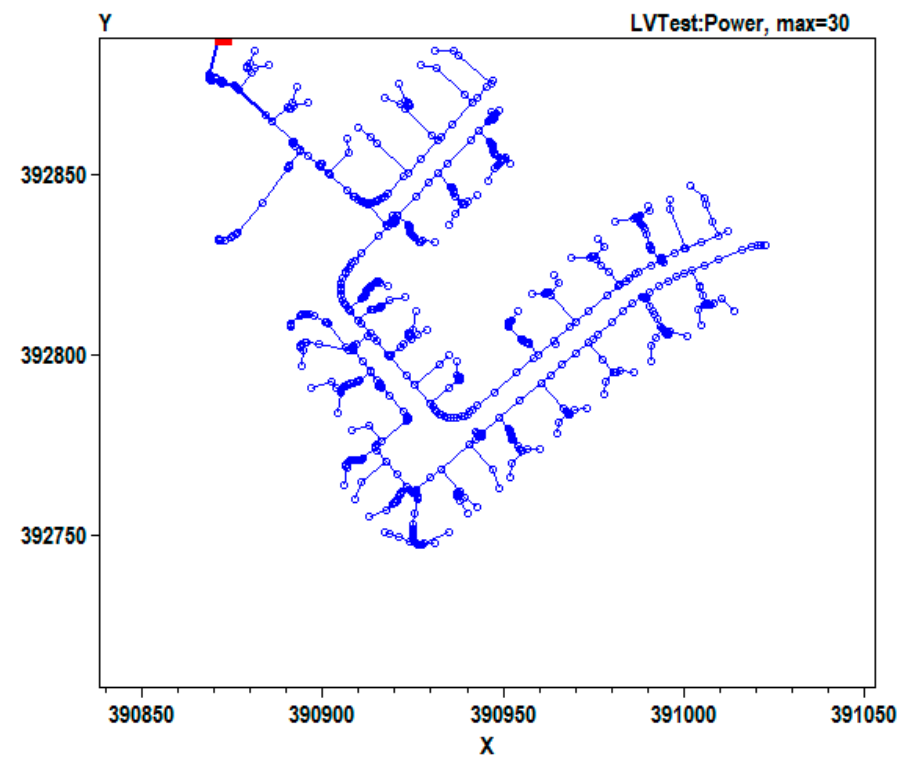

Fig. 3. The single-line diagram of low voltage (LV) test feeder [16].

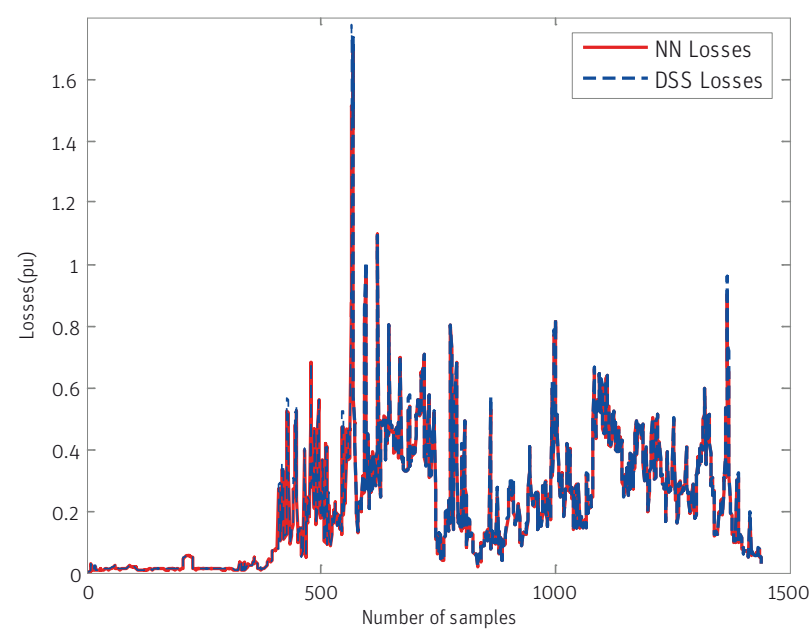

(a)

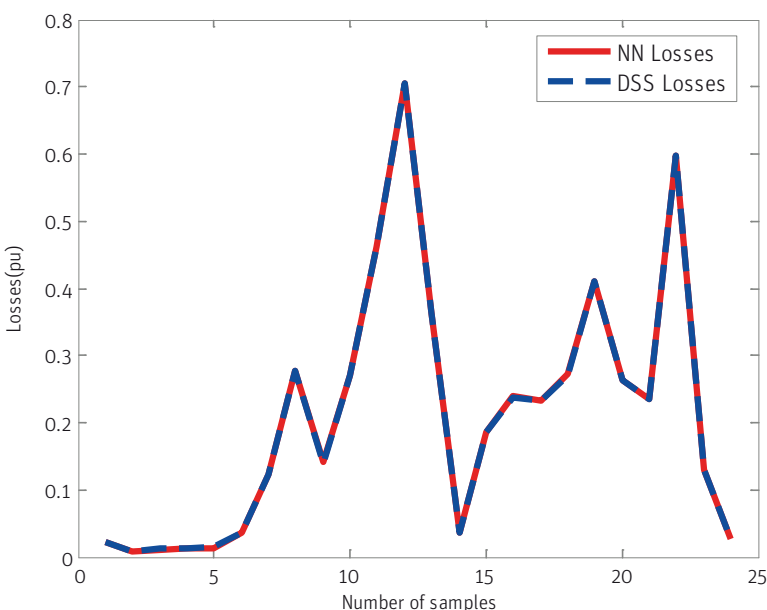

(b)

Fig. 4. The estimated losses at different time resolutions using the proposed model and OpenDSS. (a) 1 min resolution and (b) $1 \mathrm{hr}$ resolution.

To construct the offline loss model, we used a dataset of load and PV generation profiles that contains 43200 samples. For analyzing the performance of the proposed method, we have performed the following experiments:

- The power loss is analyzed for six different time resolutions for phases A, B and C, separately. The estimated losses are compared to the exact power loss of OpenDSS software.

- For accuracy validation, MSE, RMSE, MAE, MAPE, and SSE are calculated for all the three-phases.

- To highlight the computational efficiency of the proposed method, the execution time of the proposed method for estimating losses is computed and compared to the execution time of the exact approach.

\section{B. Performance Analysis}

The performance of the proposed method is compared to the exact iterative time-series power flow approach (OpenDSS) for phases A, B, and C. Specifically, we estimate the power loss profile for a day with six different time resolutions (1min, $5 \mathrm{~min}, 10 \mathrm{~min}, 15 \mathrm{~min}, 30 \mathrm{~min}$ and $1 \mathrm{hr})$. For example, the numbers of samples in datasets for the day per 1 min and $1 \mathrm{hr}$ are 1440 samples $\left(24^{*} 60\right)$ and 24 , respectively.
Fig. 4 shows the estimated losses at $1 \mathrm{~min}$ and $1 \mathrm{hr}$ resolutions using the proposed model and OpenDSS. It is evident that the estimated losses during the day for the two resolutions almost match those of the exact method. Another notice is the loss profile of $1 \mathrm{~m}$ resolutions differs from the $1 \mathrm{hr}$ resolutions, higher fluctuations appear in $1 \mathrm{~m}$ resolutions than $1 \mathrm{hr}$ resolutions. This means that the higher resolution of datasets can sufficiently represent the actual loss profiles in which the PV and load profiles have intermittent nature. However, the computational burden of the existing iterative methods will be increased when higher $\mathrm{PV}$ and load datasets are required to be analyzed. To solve this issue, the proposed method can accurately calculate the losses with large datasets (high resolution) in a very short time, thanks to the developed offline model.

Table I, Table II and Table III summarize the values of MSE, RMSE, MAE, MAPE, and SSE for phases A, B, and C, respectively at the six resolutions. It is obvious that the values of all errors are very small with respect to the exact solutions of the losses. Further, they are low at $1 \mathrm{~h}$ resolution (coarse resolution) while they are high at $1 \mathrm{~m}$ resolution (finest resolution). The lowest value of MSE appeared at $1 \mathrm{~h}$ resolution for phase A (6.1190e-07) while the largest value appears at $1 \mathrm{~m}$ resolution for phase B $(3.2222 \mathrm{e}-04)$. The same trend is noticed for 
MAE, MAPE, and SSE. For the three phases, the RE values are less than 0.05 . Note that for this test system, the estimated results of phase $A$ are more accurate than those of phase B and $\mathrm{C}$, but this is not a general rule for distribution systems.

\section{Computational Performance of the Proposed Method}

For a further description of the contribution of the proposed machine learning-based method, the execution times for solving the losses during the day with six resolutions are computed for the proposed method and exact method (OpenDSS). Table IV shows the computational times of the two methods. The execution time required for the OpenDSS is very long compared to the proposed method. For $1 \mathrm{hr}$ resolution, the OpenDSS takes approximately 1.5 sec while the proposed method takes only 0.02 seconds for obtaining the results. The execution time of OpenDSS is greatly increased with the data resolution, for example, in the case of $1 \mathrm{~min}$ resolution, the OpenDSS takes around $41 \mathrm{sec}$. However, our proposed method takes less than $0.04 \mathrm{sec}$.

\section{Comparison}

To demonstrate the performance of the NN model, we compare it with a support vector regression (SVR) model. Table V, Table VI and Table VII show the MSE, RMSE, MAE, MAPE, SSE and RE values of the SVR model for phases A, B, and C, respectively at the six resolutions. For phase A, the SVR model achieves MMSE of 0.0028 and 3.3389e-04 with $1 \mathrm{~m}$ and $1 \mathrm{~h}$ resolutions, receptively. With phase B, it gives MSE of 0.0031 and $3.8189 \mathrm{e}-04$ with $1 \mathrm{~m}$ and $1 \mathrm{~h}$ resolution, respectively. In the case of phase C, the SVR model gives a MSE less than 7e-04 with all resolutions. For the three phases, the RE values are less than 0.23 , which are much higher than those of the $\mathrm{NN}$ model ( $\mathrm{RE}$ values $<0.05$ ). In general, the comparison between the errors of the $\mathrm{NN}$ model shown in Tables I-III and the errors of the SVR model shown in Tables V-VII reveals that the NN model achieves prediction errors much lower than those of the SVR model. Therefore, it seems that the NN model is more suitable for this task.

TABLE I. MSE, RMSE, MAE, MAPE, and SSE for Phases A by the Proposed Method

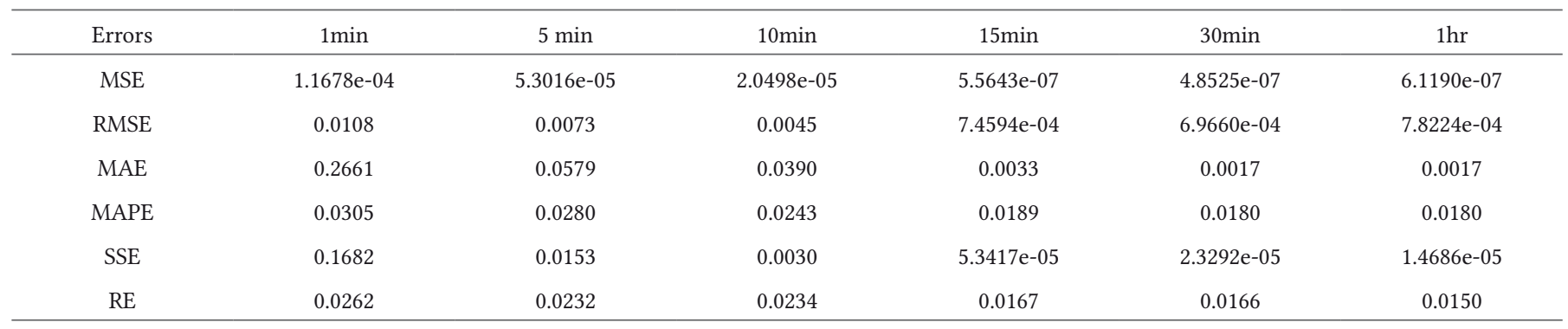

TABLE II. MSE, RMSE, MAE, MAPE, And SSE for Phases B by the Proposed Method

\begin{tabular}{ccccccc}
\hline Errors & $1 \mathrm{~min}$ & $5 \mathrm{~min}$ & $10 \mathrm{~min}$ & $15 \mathrm{~min}$ & $30 \mathrm{~min}$ & $1 \mathrm{hr}$ \\
\hline MSE & $3.2222 \mathrm{e}-04$ & $2.0802 \mathrm{e}-04$ & $1.9646 \mathrm{e}-04$ & $1.7727 \mathrm{e}-06$ & $1.5828 \mathrm{e}-06$ & 0.0013 \\
RMSE & 0.0179 & 0.0144 & 0.0140 & 0.0013 & 0.0013 \\
MAE & 0.3525 & 0.1071 & 0.1071 & 0.0048 & 0.0023 & 0.0295 \\
MAPE & 0.0504 & 0.0449 & 0.0416 & 0.0309 & 0.024 \\
SSE & 0.4640 & 0.0599 & 0.0283 & $1.7018 \mathrm{e}-04$ & 0.0280 \\
RE & 0.0435 & 0.0395 & 0.0348 & 0.0289 & $0.0276 \mathrm{e}-05$ & $2.9395 \mathrm{e}-05$ \\
\hline
\end{tabular}

TABLE III. MSE, RMSE, MAE, MAPE, ANd SSE for Phases C by the Proposed Method

\begin{tabular}{|c|c|c|c|c|c|c|}
\hline Errors & $1 \mathrm{~min}$ & $5 \mathrm{~min}$ & $10 \mathrm{~min}$ & $15 \mathrm{~min}$ & $30 \mathrm{~min}$ & $1 \mathrm{hr}$ \\
\hline MSE & $2.2134 \mathrm{e}-04$ & $1.457 \mathrm{e}-04$ & $1.0609 \mathrm{e}-04$ & $1.7281 \mathrm{e}-06$ & $1.0019 \mathrm{e}-06$ & $8.8531 \mathrm{e}-07$ \\
\hline RMSE & 0.0149 & 0.0121 & 0.0103 & 0.0013 & 0.0010 & $9.4091 \mathrm{e}-04$ \\
\hline MAPE & 0.0482 & 0.0431 & 0.0388 & 0.0294 & 0.0262 & 0.0232 \\
\hline SSE & 0.3187 & 0.0420 & 0.0153 & $1.6590 \mathrm{e}-04$ & $4.8089 \mathrm{e}-05$ & $2.1248 \mathrm{e}-05$ \\
\hline
\end{tabular}

Table iV. Computational Performance Of the Proposed Method (Time in Seconds)

\begin{tabular}{ccccccc}
\hline Method & $1 \mathrm{~min}$ & $5 \mathrm{~min}$ & $10 \mathrm{~min}$ & $15 \mathrm{~min}$ & $30 \mathrm{~min}$ & $1 \mathrm{hr}$ \\
\hline OpenDSS & 41.490263 & 7.521413 & 3.939896 & 2.685175 & 1.570552 & 0.014474 \\
NN phase A & 0.037296 & 0.018610 & 0.017173 & 0.016421 & 0.011219 & 0.012274 \\
NN phase B & 0.034559 & 0.014244 & 0.013253 & 0.010945 & 0.021032 & 0.02462 \\
NN phase C & 0.039015 & 0.014491 & 0.013141 & 0.02186 \\
\hline
\end{tabular}


TABLE V. MSE, RMSE, MAE, MAPE, AND SSE for PhASes A by SVR

\begin{tabular}{ccccccc}
\hline Errors & $1 \mathrm{~min}$ & $5 \mathrm{~min}$ & $10 \mathrm{~min}$ & $15 \mathrm{~min}$ & $30 \mathrm{~min}$ & \multicolumn{2}{c}{$1 \mathrm{hr}$} \\
MSE & 0.0028 & 0.0014 & 0.0017 & $6.3287 \mathrm{e}-04$ & $0.3239 \mathrm{e}-04$ & 0.0289 \\
RMSE & 0.0533 & 0.0370 & 0.0415 & 0.0252 & 0.1626 & 0.0183 \\
MAE & 1.1800 & 0.4249 & 0.4249 & 0.1630 & 0.0548 \\
MAPE & 0.2061 & 0.1841 & 0.1747 & 0.1528 & 0.1526 & 0.0400 \\
SSE & 4.0875 & 0.3936 & 0.2480 & 0.0608 & 0.0080 \\
RE & 0.1173 & 0.0988 & 0.0801 & 0.0927 & 0.0607 \\
\hline
\end{tabular}

TABLE VI. MSE, RMSE, MAE, MAPE, AND SSE for PhASEs B By SVR

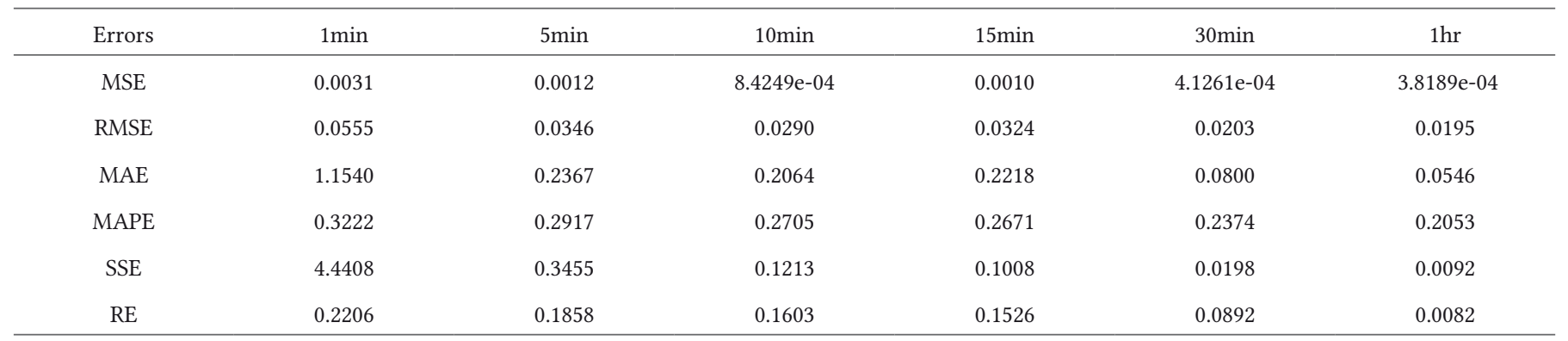

TABLE VII. MSE, RMSE, MAE, MAPE, AND SSE For PHASES C By SVR

\begin{tabular}{|c|c|c|c|c|c|c|}
\hline Errors & $1 \mathrm{~min}$ & $5 \mathrm{~min}$ & $10 \mathrm{~min}$ & $15 \mathrm{~min}$ & $30 \mathrm{~min}$ & $1 \mathrm{hr}$ \\
\hline RMSE & 0.0258 & 0.0203 & 0.0228 & 0.0176 & 0.0182 & 0.0158 \\
\hline MAE & 0.5486 & 0.1541 & 0.1541 & 0.0636 & 0.0635 & 0.0355 \\
\hline MAPE & 0.2707 & 0.2502 & 0.2382 & 0.2292 & 0.2235 & 0.2385 \\
\hline
\end{tabular}

\section{CONCLUSION}

In this paper, an efficient method has been proposed for performing time-series simulations for unbalanced power distribution systems with PV. Unlike the related iterative methods, our proposed method is based on machine learning algorithms. The proposed method has been applied to the IEEE 906 Bus European LV Test Feeder with PV gridconnected units. The proposed method is validated using OpenDSS software. The test of the proposed method has been carried out at six different resolution times $(1 \mathrm{hr}, 30 \mathrm{~min}, 15 \mathrm{~min}, 10 \mathrm{~min}, 5 \mathrm{~min}$, and $1 \mathrm{~min})$. When comparing between the trained model and OpenDSS, the calculated results have a strong matching. The calculation time required by the OpenDSS for computing losses is too long compared to the proposed method, especially at the high resolution (i.e. $1 \mathrm{~min}$ ). The experimental results show that the NN model outperforms the SVR model for time-series simulations. The results demonstrate the effectiveness of the proposed method.

The main goal of this work is to show that the application of machine learning is a promising approach to estimate energy loss in large-scale distribution systems. So, the contribution of this work is to demonstrate the applicability this approach while not trying to choose the most proper machine learning technique. Further, we believe that the results of an efficient machine learning technique can yield acceptable results for this application. The future work will be directed to consider diverse renewable energy sources, such as wind turbine generating systems.

\section{REFERENCES}

[1] Said, S. M., Aly, M., Hartmann, B., Alharbi, A. G., \& Ahmed, E. M. "SMESBased Fuzzy Logic Approach for Enhancing the Reliability of Microgrids Equipped With PV Generators," IEEE Access, vol. 7, pp. 92059-92069, 2019.

[2] Ali, Abdelfatah, D. Raisz, K. Mahmoud and M. Lehtonen. "Optimal Placement and Sizing of Uncertain PVs Considering Stochastic Nature of PEVs," IEEE Transactions on Sustainable Energy, vol. 11, no. 3, pp. 16471656, July 2020, doi: 10.1109/TSTE.2019.2935349.

[3] R. A. Verzijlbergh, L. J. De Vries, and Z. Lukszo, "Renewable Energy Sources and Responsive Demand. Do We Need Congestion Management in the Distribution Grid?," IEEE Trans. Power Syst., vol. 29, no. 5, pp. 2119-2128, Sep. 2014.

[4] V. Khare, S. Nema, and P. Baredar, "Solar-wind hybrid renewable energy system: A review," Renew. Sustain. Energy Rev., vol. 58, pp. 23-33, May 2016.

[5] X. Yang, M. Xu, S. Xu, and X. Han, "Day-ahead forecasting of photovoltaic output power with similar cloud space fusion based on incomplete historical data mining," Appl. Energy, vol. 206, pp. 683-696, Nov. 2017.

[6] M. Bhattacharya, S. R. Paramati, I. Ozturk, and S. Bhattacharya, "The effect of renewable energy consumption on economic growth: Evidence from top 38 countries," Appl. Energy, vol. 162, pp. 733-741, Jan. 2016.

[7] T. Al Momani, A. Harb, and F. Amoura, "Impact of photovoltaic systems on voltage profile and power losses of distribution networks in Jordan," in 2017 8th International Renewable Energy Congress (IREC), 2017, pp. 1-6.

[8] N. I. Zolkifri, C. K. Gan, A. Khamis, K. A. Baharin, and M. Y. Lada, "Impacts of residential solar photovoltaic systems on voltage unbalance and network losses," in TENCON 2017-2017 IEEE Region 10 Conference, 2017, pp. 2150-2155.

[9] T.-F. Wu, C.-H. Chang, Y.-D. Chang, and K.-Y. Lee, "Power loss analysis of 
grid connection photovoltaic systems," in 2009 International Conference on Power Electronics and Drive Systems (PEDS), 2009, pp. 326-331.

[10] S. Daud, A. Fazliana, A. Kadir, and C. K. Gan, S. Daud, A. F. A. Kadir and C. K. Gan, "The impacts of distributed Photovoltaic generation on power distribution networks losses," 2015 IEEE Student Conference on Research and Development (SCOReD), Kuala Lumpur, 2015, pp. 11-15, doi: 10.1109/ SCORED.2015.7449305.

[11] V. H. M. Quezada, J. R. Abbad and T. G. S. Roman, "Assessment of energy distribution losses for increasing penetration of distributed generation," in IEEE Transactions on Power Systems, vol. 21, no. 2, pp. 533-540, May 2006, doi: 10.1109/TPWRS.2006.873115.

[12] H. Le Nguyen, "Newton-Raphson method in complex form [power system load flow analysis]," in IEEE Transactions on Power Systems, vol. 12, no. 3, pp. 1355-1359, Aug. 1997, doi: 10.1109/59.630481.

[13] H. Liu and T. Feng, "Study on the Convergence of Solving Linear Equations by Gauss-Seidel and Jacobi Method," in 2015 11th International Conference on Computational Intelligence and Security (CIS), 2015, pp. $100-103$.

[14] K. Mahmoud and M. Abdel-Nasser, "Fast-yet-Accurate Energy Loss Assessment Approach for Analyzing/Sizing PV in Distribution Systems using Machine Learning," IEEE Transactions on Sustainable Energy, vol. 10, no. 3, pp. 1025-1033, July 2019, doi: 10.1109/TSTE.2018.2859036.

[15] M. Abdel-Nasser, K. Mahmoud, and H. Kashef, "A Novel Smart Grid State Estimation Method Based on Neural Networks," Int. J. Interact. Multimed. Artif. Intell., vol. 5, no. 1, p. 92, 2018.

[16] Electric Power Research Institute, OpenDSS, Distribution System Simulator [Online], Available: http://sourceforge.net/projects/ electricdss/.

[17] Gurney K. An introduction to neural networks. CRC press, 1997.

[18] O. N. A. Al-allaf, "Face Recognition System Based on Different Artificial Neural Networks Models and Training Algorithms," International Journal of Advanced Computer Science and Applications, vol. 4, no. 6, pp. 40-47, 2013.

[19] M. I. A. Lourakis, "A Brief Description of the Levenberg-Marquardt Algorithm Implemened by levmar The Levenberg-Marquardt Algorithm," Foundation of Research and Technology, vol. 4, no. 1, pp. 4-9, 2005.

[20] Abdel-Nasser, M., Mahmoud, K. "Accurate photovoltaic power forecasting models using deep LSTM-RNN," Neural Comput \& Applic vol. 31, pp. 2727-2740 (2019). https://doi.org/10.1007/s00521-017-3225-z

[21] Abdel-Nasser M, Mahmoud K, Lehtonen M. "Reliable Solar Irradiance Forecasting Approach Based on Choquet Integral and Deep LSTMs," IEEE Transactions on Industrial Informatics, 2020 doi: 10.1109/ TII.2020.2996235.

[22] IEEE PES Distribution Systems Analysis Subcommittee Radial Test Feeders [Online], Available: http://ewh.ieee.org/soc/pes/dsacom/ testfeeders.html

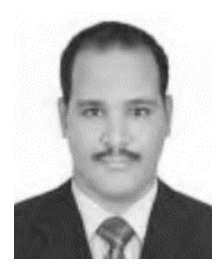

\section{Karar Mahmoud}

Karar Mahmoud received the B.S. and M.Sc. degrees in electrical engineering from Aswan University, Aswan, Egypt, in 2008 and 2012, respectively. In 2016, he received the Ph.D. degree from the Electric Power and Energy System Laboratory (EPESL), Graduate School of Engineering, Hiroshima University, Hiroshima, Japan. Since 2010, he has been with Aswan University where he is presently Assistant Professor. Currently, he is a Postdoctoral Researcher at the School of Electrical Engineering, Aalto University, Finland. His research interests include Power Systems, Renewable Energy Resources, Smart Grids, Distributed Generation, and Optimization.

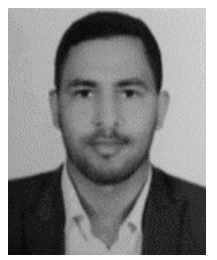

\section{Mohamed Abdel-Nasser}

Mohamed Abdel-Nasser is currently a postdoc researcher at the University Rovira i Virgili (Spain) and Assistant Professor at the Electrical Engineering Department, Aswan University (Egypt). He received his $\mathrm{PhD}$ in Computer Engineering from Universitat Rovira i Virgili (Spain) in 2016. He has published +65 papers in international journals and conferences. His research interests include the application of machine learning and deep learning to several real-world problems, smart grid analysis, and time-series forecasting.

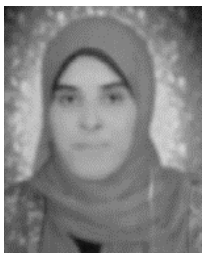

Heba Kashef

Heba Kashef received a B.Sc. degree in Electrical Engineering from the Faculty of Engineering, Aswan University, Aswan, Egypt, in 2012. She is currently working in her Msc studies the Faculty of Engineering, Aswan University, Aswan, Egypt. Her interests include machine learning, smart grid analysis, renewable energy sources.

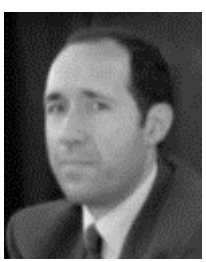

\section{Domenec Puig}

Domenec Puig received the M.S. and Ph.D. degrees in computer science from the Polytechnic University of Catalonia, Barcelona, Spain, in 1992 and 2004, respectively. In 1992, he joined the Department of Computer Science and Mathematics, Rovira i Virgili University, Tarragona, Spain, where he is currently working as a Professor since 2017. He has been the Head of the Intelligent Robotics and Computer Vision Group, Rovira i Virgili University since 2006. His research interests include artificial intelligence and mobile robotics.

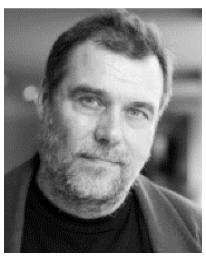

\section{Matti Lehtonen}

Matti Lehtonen received the M.Sc. and Licentiate degrees in electrical engineering from the School of Electrical Engineering, Aalto University (formerly Helsinki University of Technology), Espoo, Finland, in 1984 and 1989, respectively, and the D.Sc. degree from the Tampere University of Technology, Tampere, Finland, in 1992. Since 1987, he has been with VTT Energy, Espoo, and since 1999, he has been with the School of Electrical Engineering, Aalto University, where he is currently the Head of Power Systems and High Voltage Engineering Department. His main activities include earth fault problems, and harmonic related issues and applications of information technology in distribution automation and distribution energy management. 